\title{
Exploring the Leo II Dwarf Spheroidal: I. The Variable Star Content
}

\author{
M. H. Siegel and S. R. Majewski ${ }^{1}$ \\ University of Virginia, Department of Astronomy
}

Received __; accepted _

\footnotetext{
${ }^{1}$ David and Lucile Packard Foundation Fellow, Cottrell Scholar of the Research Corporation
} 


\begin{abstract}
We present the first comprehensive catalogue of variable stars in the Leo II dwarf spheroidal galaxy. We have identified 148 RR Lyrae type variables, of which 140 were amenable to derivation of variability parameters with our data. We have also confirmed the existence of four anomalous Cepheids as identified in previous studies.

The average period of the RR Lyrae ab variables (0.62 days), the fraction of c variables (0.24) and the minimum period of the RR Lyrae ab variables $(0.51$ days) all define Leo II as an "Oosterhoff intermediate" galaxy. We have used the properties of these variables to derive a metallicity for Leo II of approximately $[\mathrm{Fe} / \mathrm{H}]=-1.9$. We attempt to resolve discrepancies between this value and those determined by previous efforts. The presence of longer period, higher amplitude RR Lyrae variable implies a metallicity distribution that extends to as poor as $[\mathrm{Fe} / \mathrm{H}]=-2.3$.

Leo II's location on the period-metallicity relation of clusters, like that of other "Ootershoff intermediate" objects, falls between the Oosterhoff Class I and Oosterhoff Class II clusters. The properties of the variable populations of these objects are consistent with the idea that the Oosterhoff "dichotomy" is a continuum. The gap between the classes seems to be explained by the horizontal branch of Galactic globular clusters shifting away from the instability strip at at intermediate metallicities. However, Leo II, as well as other Oosterhoff intermediate objects, has a second parameter effect strong enough to leave horizontal branch stars in the instability strip.
\end{abstract}




\section{Introduction}

The Leo II dwarf spheroidal galaxy (dSph) was first identified by Harrington and Wilson (1950) on the Palomar Sky Survey. Detailed information about Leo II did not appear in the literature for over three decades due to its extreme distance and small size (except the starcount study of Hodge 1962 and abstracts on variables in Swope 1967 and Swope 1968) . A wave of interest in the 1980's (Hodge 1982; Demers and Harris 1983, hereafter DH83; Aaronson et.al. 1983; Azzopardi et al. 1985; Suntzeff et al. 1986, S86) revealed Leo II to be a metal-poor, second-parameter horizontal branch object with a handful of carbon stars.

Recently, a series of studies have expanded our knowledge of the Leo II system. Demers \& Irwin (1993, DI93) with deep BV CCD photometry, derived a metallicity of $[\mathrm{Fe} / \mathrm{H}]=$ -1.9 and revised Leo II's distance to $215 \mathrm{kpc}$. This was followed by the VI CCD studies of Lee (1995, hereafter L95) and Mighell \& Rich (1996, hereafter MR96). The former derived a metallicity of -1.97 while the latter derived a metallicity of -1.6 and determined that Leo II formed almost all of its stars between 7 and 14 Gyr ago. Both confirmed a distance modulus close to the DI93 value. Vogt et al. (1995) measured radial velocities in Leo II and derived a mass-to-light ratio of 11.1.

One of the gaps in our knowledge of Leo II remains its variable star content. The seminal work in this field was to be the comprehensive survey of Baade and Swope. Their collection of over a hundred Palomar 200-inch plates was intended to produce a complete catalogue of Leo II variables. Unfortunately, while the catalogue exists, it has not been published, nor has a rigorous analysis been applied to the data. Swope did publish two brief abstracts, one reporting the identification of 152 variables and period measurement for 76 (Swope 1967) and another reporting four anomalous Cepheids (Swope 1968). A later update by van Agt (1973) reported 64 Bailey (1902) type ab RR Lyrae stars, six type c 
and an average ab period of 0.59 days. Neither report was considered complete by the authors and neither included coordinates, finding charts or light curves. DI93 reported the identification of 80 variable candidates, but did not have enough images to fit light curves.

In this paper, we publish the first comprehensive list of positively identified variable stars in Leo II. We have found 148 RR Lyrae variables, for which we have successfully derived periods and amplitudes for 140. The distribution of periods is very similar to other dSph galaxies, with a moderate fraction of RRc variables. All of our observations are consistent with an "Oosterhoff intermediate" classification for Leo II and this classification is exhibited on a star-by-star basis. We have also identified four anomalous Cepheid variables. The presence and characteristics of these populations confirm that Leo II is metal-poor with a large intermediate age population. The longest period RRab variables hint at the existence of Leo II stars as metal-poor as $[\mathrm{Fe} / \mathrm{H}]=-2.3$.

\section{Observations and Reduction}

We observed the Leo II dSph with the 4-meter Mayall telescope at Kitt Peak on UT 8-9 April 1997 and 22-23 February 1998. Both observing runs used the T2KB $2048^{2}$ thinned CCD chip and standard Harris prescription UBV filters. Our first observing run used the old Mayall prime focus doublet corrector while our second used the new four-element corrector (now in use with the MOSAIC camera). To avoid the ghost image produced by the four-element corrector, the camera was mounted ten minutes off of the optical axis of the telescope. This increased the distortion of the images, but not to a level that was uncorrectable. All data were reduced by the standard CCDPROC pipeline in IRAF.]

\footnotetext{
${ }^{1}$ IRAF is distributed by the National Optical Astronomy Observatories, which are operated by the Association of Universities for Research in Astronomy, Inc., under
} 
All four nights suffered from very non-photometric observing conditions. We are, however, able to transform every frame to an identical instrumental system by inter-comparing individual photometric measures from frame to frame. This correction was applied iteratively until the average frame-to-frame residuals were reduced to 0.001 magnitudes. The combined instrumental magnitudes have been calibrated to the BV data set of DI93 within 0.01 magnitudes by the application of zero point and color corrections.

$$
\begin{aligned}
& m_{V}=V_{\text {inst }}-0.29-0.12(B-V)+0.12(B-V)^{2} \\
& m_{B}=B_{\text {inst }}-0.20-0.04(B-V)+0.10(B-V)^{2}
\end{aligned}
$$

This transformation does leave some non-linearity in the comparison (Figure 1). This non-linearity is approximately 0.1 magnitudes over the four magnitudes of comparison. This may be due to filter differences or a non-linearity in the CCD used in one of the two studies. Independent calibration of the data set will be clearly be required in the future. Because of this non-linearity, all variable measures and light curve determinations were made purely from the instrumental system. However, all average magnitudes and intensity-weighted mean magnitudes in our catalogues have been converted to the DI93 system via the equations listed above.

We found that a total of 72 Leo II UBV images were usable for photometry. All of these images were processed through DAOPHOT (Stetson, 1987) and ALLFRAME (Stetson, 1994). The combined photometry is complete to a depth of $B=V=24.5$ with instrumental errors at the horizontal branch of Leo II $(\mathrm{V}=22.1)$ of $\left(\sigma_{V}, \sigma_{B}\right)=(0.008,0.016)$. However, the individual images have a wide variation in quality. Their average completeness limit is $B=V=23.5$ with individual frame errors of $\left(\sigma_{V}, \sigma_{B}\right)=(0.08,0.06)$ at the horizontal branch.

cooperative agreement with the National Science Foundation. 
Accurate stellar positions were derived by using the IRAF task TFINDER with the Hubble Space Telescope Guide Star Catalogue. We were able to derive centroids for eight HST-GSC stars in our central field and used these stars to derive an approximate plate solution. Color and magnitude effects on the derived positions have not been accounted for. The coordinates listed in our variable catalogues (Tables I and III) are accurate to within approximately 0." 5. A CCD template image and the X-Y positions of the variable stars were submitted for electronic availability through the Astronomical Journal.

\section{Light Curve Fitting}

Our UBV data include $56 \mathrm{~V}$ band observations of Leo II. All but one of these were deep enough for reasonable photometry of the horizontal branch. Our observations also include thirteen usable B band observations and three usable $\mathrm{U}$ band observations, which we have excluded from the period fitting due to the very poor phase coverage of the images. A file containing the Julian dates and $\mathrm{V}$ magnitudes of all observations of our variables stars is available electronically through the Astronomical Journal.

To identify variables in our data, we used a slightly modified version of the WelchStetson (1993) variability index, adopted from that calculated in the DAOMASTER code included with ALLFRAME. We confined our variability search to V images, as they were more numerous and of better quality. Figure 2 shows the index vs. magnitude plot and our selection criteria (Index $\geq 3.0$ ). A limit of 3.0 was selected as the lowest index where most stars had clear light curves and below which significant numbers of spurious detections began. We restricted our attempts to fit light curves to stars that had at least twenty observations and had been observed in both runs.

Periods were identified by both Phase Dispersion Minimization (Stellingwerf 1978) 
and Stetson's (1996) modified version of the Lafler-Kinman index (1965). The IRAF version of PDM proved very useful for identifying true variables and revealing the nature of those variables. It was especially adept at narrowing the range within which to search for periods, at finding periods for the short-period variables, and at producing light curves. The modified LK method proved more effective with longer period stars. Once a family of reasonable periods was identified, we ran each through a $\chi^{2}$ fitting routine to refine amplitudes and to break the degeneracies of equally suitable periods. This routine used the templates of Layden (1998) for RRab stars and a sinusoidal curve for RRc.

In almost all cases, an obvious best fit was obtained. In some cases, degeneracies remained. These occured in two intervals - large degeneracies of 0.2-0.5 days and small degeneracies of 0.01-0.02 days. Large degeneracies could be broken by using the well-established differences between RRc, RRab and Cepheid stars (rise time, amplitude and color). Small degeneracies were slightly more difficult to break. We settled for the period giving the lowest $\chi^{2}$ fit. This period was then varied by several $10^{-5}$ days to further improve the fit.

The greatest deficiency of this technique is that a star that is not sampled at or near the peak brightness (within 0.1-0.2 in phase) will not be amenable to fitting. In fact, for low amplitude variables, it may not even have the contrast in brightness to be flagged as a variable. We have attempted to increase the number of detected variables by lowering the variability index threshold to 2.0. Approximately 10-20 stars in this range are potential RR Lyrae variables based on their PDM spectra. However, the magnitude contrast over the light curve is so slight that solutions prove very degenerate, if they are obtainable at all. For some of these stars, different periods have a $\chi^{2}$ value below 1.0, making them statistically equivalent. Rather than include a small number of very poor or very degenerate variables, we left any variable with an index below 3.0 out of our sample. 
Classification of variables was straight-forward. The three types of variable stars that commonly occur in dSph galaxies are RR Lyrae type ab, RR Lyrae type c and anomalous Cepheids. All three objects have well-established loci in period-amplitude-luminosityeffective temperature-rise time space.

\section{The Variable Star Catalogue}

Our variable star catalogue (Tables I and III) lists two identification numbers. The first is a running number for the variables alone, beginning with the 80 candidates from DI93. The second is a corresponding number from our master photometry catalogue of Leo II. These ID numbers are from ALLFRAME and roughly correlate with magnitude. Periods, amplitudes and pulsation modes are listed for stars for which these parameters could be derived. Magnitudes for most of the variable stars are intensity-weighted mean magnitudes. They were derived by integrating the template light curve in 0.02 phase increments (the same phase increments used in the Layden templates). For variables for which no variability parameters could be derived, the average observed magnitude is given.

We have compared our catalogue of variable stars to the list of potential variable stars in DI93. Of the 80 potential variable stars, we recover 40 as RR Lyrae type ab, 13 as type c and six as unknown RR Lyrae-like stars. One other star is an anomolous Cepheid. The remaining fifteen are not variables according to our analysis although seven of these fifteen have variability indices between 2.0 and 3.0, which places them just below our selected variable envelope; these may be true variables of very low amplitude or variables which were not in optimal phase during our observations. Table II lists the variable candidates in DI93 that did not meet our variability criterion, along with their corresponding identification number in our master photometry catalogue and our Welch-Stetson variability index. 


\section{The RR Lyrae Variables}

Figure 3 shows the amplitude-period distribution of the positively identified RR Lyrae variables in Leo II. Parameters of the variables are given in Table I while light curves are shown in Figure 4.

We note the well-established period-amplitude relationship in the type ab variables. The c variables are of nearly constant amplitude although there is some hint of the parabola-like shape predicted by Bono et al. (1997).

For eight variables with RR Lyrae-like variations we were unable to obtain satisfactory fits to their photometric data. These stars could potentially be Blazhko (1907) variables or double-mode pulsators (Cox et al. 1980; Sandage et al. 1981, hereafter SKS; Cox et al. 1983; Nemec 1985a). Our data are not sufficient for a more rigorous analysis of their light curves. We have identified an additional three variables that have a well-defined light curve in one epoch of data and a poorly defined one in the other. The likely explanation is that these are exhibiting the Blazhko effect. They are listed in Table I with the best fit that could be obtained to one epoch of photometric data; these stars are noted with a classification of "abb".

\subsection{Leo II's Stellar Populations}

Sandage (1993a, S93a) demonstrated that the shortest RRab period could be used to discern the location of the blue fundamental edge of the instability strip, which is a function of metallicity. Using his relation of

$$
\log \left(P_{a b}\right)=-0.122[\mathrm{Fe} / \mathrm{H}]-0.500
$$


and the shortest RRab period of 0.50692 days $(\log (\mathrm{P})=-0.295)$, we derive a metallicity of -1.68 on the Butler-Blanco scale (Butler 1975; Blanco 1992). This scale is 0.2 dex richer than the standard Zinn-West (1984) scale, so we correct our metallicity to -1.88.7 This is closer to the low metallicity of DI93 and other ground-based studies than the higher MR96 metallicity of $[\mathrm{Fe} / \mathrm{H}]=-1.6$. A missing variable with a period of 0.469 days would move the metallicity scale back to the MR96 value. Such a low value does not occur in any of our fitted RRab periods, nor in the degeneracies of our unfitable variables.

S93a also defined several other relations for metallicity determination, which we list in order of declining sensitivity of our data. The mean period of RRab variables is well defined by our data $(0.619 \pm 0.006$ days). S93a defines several relations between average RRab period and metallicity. We have chosen the relationships for cluster variables in which the average period has not been corrected for number density across the instability strip. This relation is use parameters closest to the actual measured quantities:

$$
\log <P_{a b}>=-0.092[\mathrm{Fe} / \mathrm{H}]-0.389
$$

This produces a Zinn-West metallicity of $-1.96 \pm 0.04$ dex. The mean period of RRc variables (0.363 \pm 0.008 days), via the relation:

$$
\log \left\langle P_{c}>=-0.119[\mathrm{Fe} / \mathrm{H}]-0.670\right.
$$

produces a Zinn-West value of $-1.93 \pm 0.08$ dex. Finally, the longest RRab period defines the red edge of the instability strip and thus the lower bound of metallicty. Our longest ab

\footnotetext{
${ }^{2}$ The Zinn-West scale has been examined by Carretta \& Gratton (1997) and Rutledge et al. (1997) and has been shown to have non-linearity in comparison to their metallicity measures. While metallicity measurements in this paper use the Zinn-West scale, the nonlinearity should have only a minor effect upon our conclusions.
} 
period is 0.8081 days. The relation:

$$
\log \left(P_{a b}\right)=-0.09[\mathrm{Fe} / \mathrm{H}]-0.280
$$

produces a -2.08 dex $(-2.28$ on the Zinn-West scale) lower bound for $[\mathrm{Fe} / \mathrm{H}]$.

The beauty of the Sandage relations is that they are completely independent of photometric zero point, reddening or calibration. Our results are consistent with previous metallicity estimates of Leo II, which have usually been around $[\mathrm{Fe} / \mathrm{H}]=-1.9$ (DH83, S86, DI93, L95).

The notable exception to these consistent metallicity determinations is MR96. Their analysis showed that photometric contamination of the red giant branch by red clump stars could have shifted DI93's estimated metallicity. However, MR96 also noted a 0.10 magnitude difference between their $V$ magnitudes and those of DI93, which they attributed to inaccurate airmass estimates on the part of DI93, possibly as a result of the eruption of Mt. Pinatubo. We are puzzled by this explanation, since DI93 observed standard stars on the same nights as Leo II. Unless the distribution of Pinatubo debris were extremely inhomogenous, it should affect the extinction terms equally for both standard and Leo II stars. The MR96 explanation would require a conspiracy of higher extinction only during Leo II observations. L95 detected a smaller $V$ band difference of 0.02 magnitudes in the brightness range of interest (both L95 and our own comparison show some non-linearity at the bright end of the DI93 CCD data). A zero point correction to the $V$ and $I$ magnitudes of MR96 might result in a shift of their color, which would produce a metallicity-reddening shift based on the MR96's use of Sarajedini's (1994) calibration technique. A shift of 0.06 in color would move the giant branch onto an $[\mathrm{Fe} / \mathrm{H}]=-1.9$ metallicity relation. In the MR96 analysis, this is a $6 \sigma$ deviation. However, Stetson (1998) has shown that WFPC2 data's best attainable photometric precision is approximately 0.02 magnitudes due to charge-transfer 
efficiency problems in the detectors. When taken in quadrature with MR96's (very small) photometric scatter and the uncertainties of the HST zero points (Holtzman et al. 1995), we estimate the uncertainties to be more likely near 0.03-0.04 magnitudes. This would reduce the shift of MR96 zero points to a $1.5-2 \sigma$ effect. Given the comparisons between the V band magnitudes of MR96, L95, DI93 and our own study, we find a systematic magnitude shift of the WFPC2 data to be a plausible reconciliation of all the metallicity measures of Leo II.

However, the existence of a more metal-rich population could be plausible if it is too young to have a significant RR Lyrae population. MR96 showed that the youngest stars in Leo II are 7 Gyr old, with a typical star having an age of 9 Gyr. This is less than what is generally thought to be the minimum age possible for an RR Lyrae star, 10-12 Gyr (Olszewski et al. 1987, O87).

It is not clear how much weight such an argument should be given. First, O87 and MR96 use different methods of analysis. O87 used the $B V$ isochrones of VandenBerg and Bell (1985) and VandenBerg (1985). MR96 averaged eight different age estimates, ranging from 7 to 11 Gyr (their Table 5). Of these, the most comparable age indicator to that used in O87 are the Revised Yale Isochrones (Green et al. 1987) which produced an age of 10 Gyr, just inside the O87 RR Lyrae minimum age envelope. Second, Leo II is only 1-3 Gyr younger than the supposed RR Lyrae minimum age, a difference comparable to the uncertainties (2 Gyr in both cases). Finally, the lower bound of RR Lyrae ages has only been determined by the comparison of Lindsay 1 to other LMC clusters and such an important result will not be conclusive until a wider variety of clusters lacking RR Lyrae's have been studied. Until the issues involved with globular cluster ages are fully resolved - and more cluster ages are determined - the possibility of a young, more metal-rich, variable-less population remains open. 
We are intrigued by the presence of ab variables with periods longer than what should be the red edge of an $[\mathrm{Fe} / \mathrm{H}]=-1.9$ population. These imply a population that is more luminous than the bulk of Leo II variables. To separate out the more luminous stars in Leo II cleanly, we have applied a period-shift analysis to our data (Sandage 1981a; Sandage 1981b; Carney et al. 1992).

Period shift is measured by comparison to a reference variable population. The usual candidate population is that of M3, for which Sandage (1982a, 1982b) derived a measure of $\Delta \log P=-\left[0.129 A_{B}+0.088+\log P\right]$, where $A_{B}$ is the amplitude in the $B$ passband. However, we applied this formulation to the recent measurements of M3 variable periods and amplitudes by Caretta et al. (1999) and Kałuz̀ny et al. (1998) and found a period shift of 0.015 by the Sandage formulation. We are unable to explain this discrepancy but have corrected the period shift zero point to produce a $\Delta \log P$ of 0.000 for the new M3 data. We have also derived a conversion ratio of $A_{B} / A_{V}=1.21$. The resulting revised period-shift relations are:

$$
\begin{aligned}
& \Delta \log P=-\left[0.129 A_{B}+0.112+\log P\right] \\
& \Delta \log P=-\left[0.156 A_{V}+0.112+\log P\right]
\end{aligned}
$$

Our $\Delta \log P$ values for Leo II from these relations are plotted in Figure 5.

The pulsation equation of Van Aldada \& Baker (1971) is:

$$
\log P_{o}=-1.772-0.68 \log \left(\frac{M}{M_{\odot}}\right)+0.84 \log \left(\frac{L}{L_{\odot}}\right)+3.48 \log \left(\frac{6500}{T_{e f f}}\right)
$$

Assuming a uniform mass, one can measure changes in luminosity by measuring the period and effective temperature of each star. Effective temperature can not be directly measured, but SKS showed that amplitude, specifically in the blue passband, can be used to measure effective temperature. The most recent revision of this relation is by Catelan (1998): 


$$
\frac{5040}{T_{e q}}=0.868-0.084 A_{B}+0.005[F e / H]
$$

Where $T_{e q}$ is the equilibrium temperature. Equilibrium temperature is not the same as effective temperature, but is very similar (Carney et al. 1992). This relation has only a weak dependence upon metallicity. By comparing the periods of two stars with identical amplitudes, we compare stars at identical temperatures. This enables us to measure differences in luminosity.

The resultant theoretical $\Delta M_{V}$ scale is on the right ordinate of Figure 5 . While there is a large amount of scatter intrinsic to the period-shift measure (and our conversion of the relations to the $V$ passband only amplifies this scatter) it is clear that the stars in Leo II are dominated by a population 0.08 magnitudes more luminous than the stars of M3 with a higher luminosity tail in the longer period variables.

To confirm the increased luminosity for the longer period variables, we have directly compared the apparent magnitudes of our higher period shift population to the bulk population. We find that the nine most shifted stars $(\Delta \log P<-.08)$ in Figure 5 (excluding the two that clearly deviate from the main magnitude locus in Figure 8) average $0.09 \pm .03$ magnitudes brighter than the bulk of the RR Lyrae stars. This is smaller than the 0.2 magnitude difference implied by the period-shift analysis (see Figure 5), which indicates a difference in mass for the more extreme Leo II variables.

If we abandon the assumption of fixed mass and use differences in magnitude to constrain luminosity differences, we can measure the relative mass of each star by rewriting the pulsation equation as:

$$
-0.68 \log \frac{M_{a v g}}{M}=-0.336\left(m-m_{\text {avg }}\right)+\Delta \log P-<\Delta \log P>
$$

where $M_{\text {avg }}$ is the average RR Lyrae mass, $M$ is the mass of an individual star, $m_{\text {avg }}$ and $m$ are the average and individual apparent magnitudes, and $\langle\Delta \log P\rangle$ is the average period 
shift in Leo II. Using this formulation, we estimate the second population of stars to be $13 \%$ less massive than the average Leo II RRab star.

While longer period variables can occur in the absence of metallicity effects (Lee \& Carney, 1999a; Pritzl et al. 1999), this signature is also seen in Sculptor (Kałuz̀ny et al. 1995, hereafter K95), which has a well-established spread in metallicity (Norris \& Bessell 1978; Smith \& Dopita 1983; Da Costa 1984; Da Costa 1988; K95; Majewski et al. 1999, hereafter M99; Hurley-Keller et al. 1999). This may warrant a closer look at the Leo II color-magnitude diagram to see if a dual metallicity model may be more appropriate, as in the case of Sculptor. While dramatic conclusions should not be drawn from a handful of unusual stars, if the increased luminosity is the product of metallicity alone, this second Leo II population would have a metallicity of $[\mathrm{Fe} / \mathrm{H}]=-2.3$ using the RR Lyrae luminosity calibration of Sandage (1993b).

\subsection{Oosterhoff Classification}

It has long been known that Galactic clusters fall into two categories based upon the properties of their RR Lyrae variables (Oosterhoff 1939; see also a history of this

phenomenon in S93a and Smith 1995). The ratio of c to ab variables of 0.24, the average RRab period of 0.62 days, and the minimum RRab period of 0.51 days are hallmarks of an Oosterhoff classification for Leo II that is intermediate between OoI and OoII. This classification is also seen in the Sculptor dSph (K95), Sextans (Mateo et al. 1995), several Magellanic clusters (Bono et al. 1994) and possibly the Draco (Nemec 1985b), Carina (Saha et al. 1986) and Ursa Minor (Nemec et al. 1988) dSph's.

S93a argued that the Oosterhoff dichotomy is the result of a continuous change of RR Lyrae properties with metallicity. The gap between Oosterhoff classes would result from 
Galactic clusters with metallicites between OoI and OoII having extreme blue horizontal branches that depopulate the instability strip (Renzini 1983; Castellani 1983). Leo II and other Oosterhoff objects, however, have a second parameter effect that populates the red end of the horizontal branch and the instability strip. Figure 6 plots the average ab periods and spectroscopic metallicities of the clusters listed in S93a and the intermediate Oosterhoff clusters and dSph's listed above. The Oosterhoff imtermediate objects fill in the gap between the Oosterhoff classes and fall close to the relation of S93a. This would support the Renzini-Castellani-Sandage explanation of the Oosterhoff dichotomy.

The one caveat to this conclusion is that the dwarf spheroidal galaxies show evidence of multiple populations (see a summary in Grebel 1997, Grebel 1998 and Mateo 1998). This may be reflected in the variables of Leo II as well as those of Sculptor, which has a metallicity distribution from $[\mathrm{Fe} / \mathrm{H}]=-1.5$ to -2.3 (Kałuz̀ny et al. 1995; M99). In principle, one could revive the Oosterhoff dichotomy by supposing that Oosterhoff intermediate objects merely reflect a superposition of OoI and OoII populations.

We find this to be a dubious interpretation. In the first place, the four intermediate LMC clusters all clearly have single populations. Second, in the case of Leo II and Sculptor, the consistency between non-variable measures of metallicity and the S93a formulation for the RR Lyrae stars indicates that the less metal-poor population is the origin of the bulk of the variables.

A third argument against this can be seen in Figure 5. The period shift is also a useful measure of the Oosterhoff effect in individual stars. Both cluster stars and field stars exhibit the Oosterhoff dichotomy by avoiding $\Delta \log P$ values between -0.01 and -0.05 (Suntzeff et al. 1991). If the Oosterhoff intermediate objects are a superposition of OoI and OoII, the mean $\Delta \log P$ value may be in the forbidden zone but individual stars should avoid it. Clearly, just as the Oosterhoff dichotomy is exhibited for Galactic cluster and field stars on 
a star-by-star basis, so do the variables of Leo II violate that dichotomy on a star-by-star basis. Our reformulation of the $\Delta \log P$ measure places 56 of our 106 RR Lyrae ab variables into the forbidden region. Using the classical $\Delta \log P$ formulation places 39 of our stars in this region. [

This by no means implies that metallicity is the only parameter that affects the bulk and individual properties of cluster RR Lyrae stars. Metallicity has been suggested as the primary factor because of the likely increase in RR Lyrae luminosity with declining metallicity, which would produce the Oosterhoff effect (SKS). Any other effect that increases RR Lyrae luminosity, including HB evolution, would also affect the properties of the variable stars (Lee, Demarque \& Zinn 1990, LDZ). Evolution is apparently the best explanation for the deviation of M2 from the primary locus in Figure 6 (Lee \& Carney 1999b). However, Figure 6 shows that metallicity is the "first parameter" of average RRab period.

\subsection{Distance Modulus}

RR Lyrae variables are a useful tool for measuring distance modulus. However, the only studies that could be used to calibrate our data to true apparent magnitudes (DH83, DI93, L95, MR96) also use the horizontal branch as a distance indicator. Adapting these other studies to calibrate our RR Lyrae mean magnitudes for the same purpose would be circular and pointless. However, if used in combination with other calibration techniques, our RR Lyrae variable photometry might eventually serve to refine Leo II's distance measure.

More importantly, the metallicity spread in the RR Lyrae variables of the dSph galaxies could assist with the refinement of the $M_{V}-[\mathrm{Fe} / \mathrm{H}]$ relation. Similar efforts with the

\footnotetext{
${ }^{3}$ An identical argument is used by Mateo et al. (1995) to show that the RRab stars of Sextans are Oosterhoff intermediate.
} 
globular cluster $\omega$ Centauri (Dickens 1989) have been inconclusive, possibly because of the complicating effects of evolution (Gratton et al. 1986; Lee 1991). It has been argued that there is no universal $M_{V}-[\mathrm{Fe} / \mathrm{H}]$ relation that is independent of the effect of evolution as expressed in horizontal branch morphology (LDZ; Clement \& Shelton 1999; Lee \& Carney 1999a; Demarque et al. 1999). Untangling the evolution-metallicity-absolute magnitude

question, especially in the absence of direct spectroscopic measures of RR Lyrae metallicity, is well beyond the scope of this paper.

\section{Anomalous Cepheids}

Swope (1968) identified four anomalous Cepheid variables in Leo II. We have also identified four variables with parameters similar to Swope's that are cleanly separated from the RR Lyrae locus in period-amplitude-magnitude-color-rise time space. Light curves and derived parameters are given in Figure 7 and Table III respectively. We have listed what we believe to be the correct cross-identificiations to the anomalous Cepheids of the Swope study. However, her V1 and V51 are not clearly distinguishable in the absence of a finding chart.

The apparent magnitudes and periods of all RR Lyrae and Cepheid variables are plotted in Figure 7 with the fundamental and first overtone Cepheid pulsation lines from Nemec et al. (1994). We find three fundamental and one first overtone pulsators among the anomalous Cepheids.

The origin of anomalous cepheids and their implications are still poorly understood (c.f. Nemec et al. 1988; Mateo et al. 1995). They can be indicative of a 5-10 Gyr old population, which MR96 showed exists in Leo II. They can also be mass-transfer binaries, for which a specific ratio of $\sim 1-10$ for blue-stragglers to anomalous Cepheids is predicted (Renzini, 
Mengel \& Sweigart 1977). Our photometry of Leo II, at present, does not allow a robust estimate of the blue straggler content due to the rapidly decling photometric accuracy as the data approach the main-sequence turnoff. However, if the suggested ratio were to hold up,

the deep photometry of MR96 would be expected to show 0.5-5 blue stragglers (normalizing to the ratio of horizontal branch stars in our study and MR96). Even a cursory glance at their Figure 4 reveals a much higher number of blue stragglers. However, it is unclear as to how centrally concentrated these objects are (the MR96 pointing is close to the center of Leo II). Only deep wide-field photometry of the entire dSph will give an accurate statistical handle on this question.

\section{Conclusions}

\subsection{Leo II and the Oosterhoff Continuum}

The RR Lyrae variables in Leo II place its dominant metallicity at $[\mathrm{Fe} / \mathrm{H}]=-1.9$. A handful of large $\Delta \log P$ stars appears to imply a lower metallicity population. This is not conclusive, but warrants a more comprehensive look at the color-magnitude diagram of Leo II, which we will perform in a future contribution. At present, we can make no contribution regarding Leo II's distance modulus due to the non-photometric conditions during our observations.

Leo II is Oosterhoff intermediate class, like several other objects, including a number of dSph galaxies. Its violation of the Oosterhoff gap is exhibited in both the bulk and individual properties of its RR Lyrae stars. The Oosterhoff intermediate objects fill in the Oosterhoff gap, and therefore support the interpretation that metallicity is the dominant parameter in determining average RRab period. 


\subsection{The Fornax-Leo-Sculptor Stream Revisited}

Lynden-Bell (1982) was the first to note that the dSph galaxies appear to lie in two great streams in the sky. One of these streams appears to include the Fornax, Leo I, Leo II and Sculptor dSph galaxies. Majewski (1992) noted that Phoenix, Sextans and several second parameter objects also lie along this plane. Palma et al. (2000) found that the outermost $\left(R_{G C}>25 \mathrm{kpc}\right)$ second parameter globular clusters have the highest probability of being aligned with one of the two streams. While such alignments could be coincidental, they could also result from a common formation mechanism, in which case one might expect some similiarities in the stellar populations of brethren objects.

Comparisons between the populations of Leo II and Sculptor are possible from the available data. Both show a strong low metallicity $([\mathrm{Fe} / \mathrm{H}]=-1.7$ in Sculptor), second parameter, Oosterhoff intermediate population. Both also show evidence of a less populous, even more metal-poor population. In Sculptor, this population does not have a second parameter effect and is spatially dispersed (M99). The presence of a spatial gradient in the horizontal morphology of Leo II (Da Costa et al. 1996) may indicate a similar abundance-HB morphology pattern to that observed in Sculptor.

Fornax also has multiple populations. While it is dominated by a population at $[\mathrm{Fe} / \mathrm{H}]=-1.5$, it shows evidence of populations from -0.7 to -2.2 in both its field star and

globular cluster population (c.f. Buonanno et al. 1985; Beauchamp et al. 1995). The most metal-poor population, like that in Sculptor, is much more extended than the metal-rich populations (Grebel \& Stetson 1998). One could envision a scenario in which Fornax, Leo and Sculptor all originated in a common $[\mathrm{Fe} / \mathrm{H}] \sim-2.3$, first parameter HB progenitor, then, after disociation, each object followed it own star formation history. Alternatively, perhaps there is something fundamental about the "threshold" metallicity $[\mathrm{Fe} / \mathrm{H}]=-2.3$ in small stellar systems. We note that the lowest metallicities in the Milky Way globular cluster 
system are also around $[\mathrm{Fe} / \mathrm{H}]=-2.3$ (Harris 1996).

Any firm conclusions about correlations between Leo II, Sculptor and Fornax must await the measurement of their absolute proper motions. This will be the single most powerful discriminant for or against a common history. However, the similarities between the most metal-poor populations in each of Leo II, Sculptor and Fornax are intriguing.

The authors would like to thank Marcio Catelan for reading this article before submission and providing many useful comments. We would also like to thank the anonymous referee for useful remarks. MHS was provided thesis travel support by NOAO. MHS and SRM were supported by awards from the David and Lucile Packard Foundation and the Research Corporation, as well as National Science Foundation CAREER Award grant, AST-9702521. 


\section{REFERENCES}

Aaronson, M., Olszewski, E. W. \& Hodge, P. W. 1983, ApJ, 267, 271

Azzopardi, M., Lequeux, J. \& Westerlund, B. E. 1985, A\&A, 144, 388

Bailey, S. I. 1902, Annals of Harvard College Observatory, 38, 1

Beauchamp, D., Hardy, E., Suntzeff, N. B. \& Zinn, R. 1995, AJ, 109, 1628

Blanco, V. 1992, AJ, 104, 734

Blazhko, S. 1907, Astron. Nachr., 175, 325

Bono, G., Caputo, F. \& Stellingwerf, R.F. 1994, ApJ, 423, 294

Bono, G., Caputo, F., Castellani, V. \& Marconi, M. 1997, A\&AS, 121, 327.

Buonanno, R., Corsi, C. E., Fusi Pecci, F., Hardy, E. \& Zinn, R. 1985, A\&A, 152, 65

Butler, D. 1975, ApJ, 200, 68

Carney, B. W., Storm, J. \& Jones, R. V. 1992, ApJ, 386, 663

Carretta, E. \& Gratton, R. G. 1997, A\&AS, 121, 95

Carretta, E., Cacciari, C., Ferraro, F. R., Fusi Pecci, F. \& Tessicini, G. 1998, MNRAS, 298, 1005

Castellani, V. 1983, MSAIt, 54, 141

Catelan, M. 1998, ApJ, 495, L81

Clement, C. M. \& Shelton, I. 1999, ApJ, 515, L85

Cox, A. N., King, D. S. \& Hodson, S. W. 1980, ApJ, 236, 219

Cox, A. N., Hodson, S. W. \& Clancy, S. P. 1983, ApJ, 266, 94

Da Costa, G. S. 1984, ApJ, 285, 483 (D84) 
Da Costa, G. S. 1988, in IAU Symp. 126, The Harlow Shapley Symposium on Globular Cluster Systems in Galaxies, eds. J. E. Grindlay \& A. G. D. Philip (Dordrecht: Kluwer), 217

Da Costa, G. S., Armandroff, T. E., Caldwell, N. \& Seitzer, P. 1996, AJ, 112, 2576

Demarque, P., Zinn, R., Lee, Y. W. \& Yi, S. 1999, ApJ, accepted

Demers, S. \& Harris, W. E. 1983, AJ, 88, 329 [DH83]

Demers, S. \& Irwin, M. J. 1993, MNRAS, 261, 657 [DI93]

Dickens, R. J. 1989, in The Use of Pulsating Stars in Fundamental Problems in Astronomy, ed. E. G. Schmidt, (Cambridge: Cambridge University Press), p. 141

Gratton, R. G., Tornambe, A. \& Ortolani, S. 1986, A\&A, 169, 111

Grebel, E. K. 1997, RvMA, 10, 29

Grebel, E. K. 1998, in IAU Symposium 192: The Stellar Content of Local Group Galaxies, eds. P. Whitelock \& R. Cannon, ASP Conf. Ser. Vol 192, (San Francisco: ASP), p. 1

Grebel, E. K. \& Stetson, P. B. 1998, in IAU Symposium 192: The Stellar Content of Local Group Galaxies, eds. P. Whitelock \& R. Cannon, ASP Conf. Ser. Vol 192, (San Francisco: ASP), p. 11

Green, E. M., Demarque, P. \& King, C. R. 1987, The Revised Yale Isochrones and Luminosity Functions (Yale University Observatory, New Haven)

Harrington, R. G. \& Wilson, A. G. 1950, PASP, 62, 118

Harris, W.E. 1996, AJ, 112, 1487

Hodge, P. W. 1962, AJ, 67, 125

Hodge, P. W. 1982, AJ, 87, 1668 
Holtzman, J. A., Burrows, C. J., Casertano, S., Hester, J. J., Trauger, J. T., Watson, A. M. \& Worthey, G. 1995, PASP, 107, 1065

Hurley-Keller, D. , Mateo, M. \& Grebel, E. K. 1999, ApJ, 523, L25

Kałuz̀ny, J., Kubiak, M., Szymanśki, M., Udalski, A., Krzeminśki, W. \& Mateo, M. 1995, A\&AS, 112, $407[\mathrm{~K} 95]$

Kałuz̀ny, J., Hilditch, R. W., Clement, C. \& Rucinski, S. M. 1998, MNRAS, 296, 347

Lafler, J. \& Kinman, T. D. 1965, ApJS, 11, 216

Layden, A. C. 1998, AJ, 115, 193

Lee, J. W. \& Carney, B. W. 1999a, AJ, 118, 1373

Lee, J. W. \& Carney, B. W. 1999b, AJ, 117, 2868

Lee, Y. W., Demarque, P. \& Zinn, R. 1990, ApJ, 350, 155 [LDZ]

Lee, Y. W. 1991, ApJ, 373, L43

Lee, M. G. 1995, AJ, 110, 1155 [L95]

Lynden-Bell, D. 1982, Observatory, 102, 202

Majewski, S. R., 1992, ApJS, 78, 87

Majewski, S. R., Siegel, M. H., Patterson, R. J. \& Rood, R. T. 1999, ApJ, 520, L33 [M99]

Mateo, M. 1998, ARA\&A, 36, 435

Mateo, M., Fischer, P. \& Krzeminski, W. 1995, AJ, 110, 2166

Mighell, K. J. \& Rich, M. R. 1996, AJ, 111, 777 [MR96]

Nemec, J. M. 1985a, AJ, 90, 240

Nemec, J. M. 1985b, AJ, 90, 204

Nemec, J. M., Wehlau, A. \& de Oliveira, C.M. 1988, AJ, 96, 528 
Nemec, J. M., Nemec, A. F. L. \& Lutz, T. E. 1994, AJ, 108, 222

Norris, J. \& Bessell, M. S. 1978, ApJ, 225, L49

Olszewski, E. W., Aaronson, M. \& Schommer, R. A. 1987, AJ, 93, 565

Oosterhoff, P. Th. 1939, Observatory, 62, 104

Palma, C., Majewski, S. R. \& Johnston, K. V. 1999, ApJ, submitted

Pritzl, B., Smith, H.A., Catelan, M. \& Sweigart, A. V. 1999, ApJ, accepted

Renzini, A., Mengel, J. G. \& Sweigart, A. V. 1977, A\&A, 56, 369

Renzini, A. 1983, MSAIt, 54, 335

Rutledge, G. A., Hesser, J. E. \& Stetson, P. B. 1997, /pasp, 109, 907

Saha, A., Monet, D. G. \& Seitzer, P. 1986, AJ, 92, 302

Sandage, A., Katem, B. \& Sandage, M. 1981, ApJS, 46, 41 [SKS]

Sandage, A. 1981a, ApJ, 244, L23

Sandage, A. 1981b, ApJ, 248, 161

Sandage, A. 1982a, ApJ, 252, 553

Sandage, A. 1982b, ApJ, 252, 574

Sandage, A. 1993a, AJ, 106, 687 [S93a]

Sandage, A. 1993b, AJ, 106, 703

Sarajedini, A. 1994, AJ, 107, 618

Smith, H. S. \& Dopita, M. A. 1983, ApJ, 271, 113

Smith, H. A. 1995, RR Lyrae Stars (Cambridge: Cambridge University Press)

Stellingwerf, R. F. 1978, ApJ, 224, 953

Stetson, P. B. 1987, PASP, 99, 191 
Stetson, P. B. 1994, PASP, 106, 250

Stetson, P. B. 1996, PASP, 108, 851

Stetson, P. B. 1998, PASP, 110, 1448

Suntzeff, N. B., Aaronson, M., Olszewski, E. W. \& Cook, K.H. 1986, AJ, 91, 1091 [S86]

Suntzeff, N. B., Kinman, T. D. \& Kraft, R. P. 1991, ApJ, 367, 528

Swope, H. H. 1967, PASP, 79, 439

Swope, H. H. 1968, AJ, 73, S204

van Agt, S. 1973, in Variable Stars in Globular Clusters and Related Systems, ed. J. D. Fernie, (Dordrecht: Reidel), p. 35

van Albada, T.S. \& Baker, N. 1971, ApJ, 169, 311

VandenBerg, D. A. \& Bell, R. 1985, ApJS, 58, 561

VandenBerg, D. A. 1985, ApJS, 58, 711

Vogt, S. S., Mateo, M., Olszewski, E. W. \& Keane, M. J. 1995, AJ, 109, 151

Welch, D. L. \& Stetson, P. B. 1993, AJ, 105, 1813

Zinn, R. \& West, M. J. 1984, ApJS, 55, 45

Fig. 1.- Comparison of stars from DI93 to the corrected $B$ and $V$ magnitudes of this study. While the comparisons have RMS values of 0.01 magnitudes, both show a degree of non-linearity in comparison.

Fig. 2.- $V$ magnitude plotted against the modified Welch-Stetson index. All objects above the dashed line were marked as potential variables. While the bright end locus moves above this line, almost all of these objects had increase indices due to saturation effects. 
Fig. 3.- Period-amplitude distribution of the variables in Leo II. Boxes are RRab variables, circles are RRc, filled triangles are anomalous Cepheids. Periods are in days.

Fig. 4.- RR Lyrae light curves in order of increasing period.

Fig. 5.- The period shift effect in the variables of Leo II. $\Delta \log P$ is the period shift. The right ordinate shows the corresponding magnitude shift, under the assumption of constant mass. The dashed lines mark the zone avoided by Galactic and cluster stars.

Fig. 6.- Average RRab period against spectroscopic metallicity for globular clusters and dwarf spheroidal galaxies with 10 or more measured RRab variables. Circles are OoI clusters, open triangles Oo intermediate and squares OoII. For this plot, we have adopted Draco as Oo intermediate, Carina and Ursa Minor as OoII. The solid line is the observational fit from Sandage (1993a). The significantly deviant point is M2, based on the study of Lee \& Carney (1999b).

Fig. 7.- Light curves of the anomalous Cepheid variables in Leo II.

Fig. 8.- Apparent magnitudes of all variables stars within Leo II as a function of period. RRab variables are marked with squares, RRc with circles and anomalous Cepheids with filled triangles. The solid lines are the fundamental and first overtone modes produced by Nemec et al. (1994).

TABLE I. RR Lyrae Variables 


\begin{tabular}{|c|c|c|c|c|c|c|c|c|}
\hline Var ID & Star ID & $\begin{array}{c}\text { RA } \\
\text { J2000.0 }\end{array}$ & $\begin{array}{c}\text { DEC } \\
\text { J2000.0 }\end{array}$ & $\begin{array}{l}\text { Period } \\
\text { (Days) }\end{array}$ & $A_{V}$ & $m_{V}$ & $\begin{array}{l}\text { Bailey } \\
\text { Type }\end{array}$ & $\Delta \log P$ \\
\hline V1 & 2350 & $11: 13: 23.36$ & $22: 12: 56.5$ & 0.67352 & 0.723 & 22.08 & $a b$ & -0.053 \\
\hline $\mathrm{V} 2$ & 2755 & $11: 13: 32.25$ & $22: 12: 13.1$ & 0.62137 & 0.622 & 22.17 & $\mathrm{ab}$ & -0.002 \\
\hline V5 & 4035 & $11: 13: 39.17$ & $22: 11: 21.7$ & 0.57152 & 0.967 & 22.20 & $a b$ & -0.020 \\
\hline V6 & 2597 & $11: 13: 42.30$ & $22: 10: 58.6$ & 0.64544 & 0.604 & 22.09 & $a b$ & -0.016 \\
\hline $\mathrm{V} 8$ & 2768 & $11: 13: 32.68$ & $22: 11: 29.5$ & 0.63715 & 0.757 & 22.17 & $a b$ & -0.034 \\
\hline V9 & 2153 & $11: 13: 27.92$ & $22: 11: 43.3$ & 0.62357 & 0.751 & 21.89 & $a b$ & -0.024 \\
\hline V10 & 3149 & $11: 13: 20.75$ & 22:12:02.0 & 0.32033 & 0.769 & 22.13 & $\mathrm{c}$ & \\
\hline V11 & 3373 & $11: 13: 29.65$ & $22: 11: 23.4$ & 0.65546 & 0.619 & 22.24 & $a b$ & -0.025 \\
\hline V14 & 3735 & $11: 13: 25.80$ & $22: 11: 38.0$ & 0.64419 & 0.535 & 22.21 & $a b$ & -0.004 \\
\hline V15 & 2059 & $11: 13: 31.82$ & $22: 11: 10.6$ & 0.62126 & 0.997 & 22.16 & $a b$ & -0.061 \\
\hline V16 & 1892 & $11: 13: 35.79$ & $22: 10: 53.0$ & 0.39198 & 0.386 & 21.71 & $\mathrm{c}$ & \\
\hline V17 & 3262 & 11:13:41.16 & $22: 10: 18.9$ & 0.56056 & 1.040 & 22.36 & $a b$ & -0.023 \\
\hline V18 & 2379 & 11:13:19.63 & $22: 11: 46.2$ & 0.79279 & 0.571 & 22.09 & $a b$ & -0.100 \\
\hline V19 & 3316 & $11: 13: 34.28$ & $22: 10: 37.0$ & 0.40336 & 0.694 & 22.13 & $\mathrm{c}$ & \\
\hline V21 & 3217 & $11: 13: 38.10$ & $22: 10: 11.8$ & 0.65821 & 0.633 & 22.18 & $a b$ & -0.029 \\
\hline $\mathrm{V} 22$ & 1965 & $11: 13: 16.90$ & $22: 11: 36.6$ & 0.59125 & 1.017 & 22.08 & $\mathrm{ab}$ & -0.042 \\
\hline $\mathrm{V} 23$ & 3144 & $11: 13: 41.58$ & $22: 09: 40.3$ & 0.53865 & 1.344 & 22.31 & $a b$ & -0.053 \\
\hline V24 & 4297 & $11: 13: 24.46$ & $22: 10: 53.0$ & 0.39538 & 0.636 & 22.28 & $\mathrm{c}$ & \\
\hline $\mathrm{V} 25$ & 3068 & $11: 13: 20.36$ & $22: 11: 10.3$ & 0.38665 & 0.547 & 22.27 & $\mathrm{c}$ & \\
\hline V28 & 3193 & $11: 13: 32.64$ & 22:09:33.2 & & & 22.10 & unk & \\
\hline V29 & 2451 & $11: 13: 32.27$ & $22: 09: 27.8$ & 0.76034 & 0.747 & 22.18 & $a b$ & -0.110 \\
\hline V30 & 3230 & 11:13:30.71 & $22: 09: 17.3$ & 0.57145 & 0.776 & 22.28 & $a b$ & 0.010 \\
\hline V31 & 2676 & $11: 13: 35.88$ & $22: 08: 47.6$ & 0.36563 & 0.778 & 22.18 & $\mathrm{c}$ & \\
\hline V32 & 3992 & 11:13:19.63 & $22: 09: 55.9$ & 0.38714 & 0.502 & 22.27 & $\mathrm{c}$ & \\
\hline V33 & 3273 & 11:13:28.22 & 22:09:17.8 & 0.63122 & 0.743 & 22.26 & $a b$ & -0.028 \\
\hline V34 & 2472 & 11:13:18.82 & $22: 09: 57.0$ & 0.62329 & 0.643 & 22.05 & $a b$ & -0.007 \\
\hline
\end{tabular}


TABLE I. Continued. RR Lyrae Variables 


\begin{tabular}{|c|c|c|c|c|c|c|c|c|}
\hline Var ID & Star ID & $\begin{array}{c}\mathrm{RA} \\
\mathrm{J} 2000.0\end{array}$ & $\begin{array}{c}\text { DEC } \\
\text { J2000.0 }\end{array}$ & $\begin{array}{l}\text { Period } \\
\text { (Days) }\end{array}$ & $A_{V}$ & $m_{V}$ & $\begin{array}{l}\text { Bailey } \\
\text { Type }\end{array}$ & $\Delta \log P$ \\
\hline V36 & 2770 & $11: 13: 26.43$ & 22:09:14.0 & 0.61411 & 0.683 & 22.21 & $a b$ & -0.007 \\
\hline V37 & 4140 & $11: 13: 26.46$ & 22:09:11.3 & 0.35721 & 0.497 & 22.28 & $\mathrm{c}$ & \\
\hline V38 & 3306 & 11:13:27.06 & 22:09:05.0 & 0.26012 & 0.489 & 22.16 & $\mathrm{c}$ & \\
\hline V39 & 3597 & 11:13:33.00 & 22:08:35.9 & 0.61989 & 0.450 & 22.20 & $a b$ & 0.025 \\
\hline V40 & 2863 & 11:13:18.29 & $22: 09: 38.4$ & 0.70926 & 0.510 & 22.01 & $a b$ & -0.042 \\
\hline V41 & 2598 & $11: 13: 29.06$ & $22: 08: 48.6$ & 0.59113 & 0.993 & 22.04 & $a b$ & -0.039 \\
\hline V43 & 3018 & 11:13:32.61 & $22: 08: 17.2$ & 0.56856 & 0.758 & 22.23 & $a b$ & 0.015 \\
\hline V44 & 3092 & $11: 13: 34.95$ & $22: 07: 59.2$ & 0.36896 & 0.559 & 22.16 & $\mathrm{c}$ & \\
\hline V46 & 4203 & $11: 13: 30.18$ & $22: 08: 17.0$ & 0.60083 & 0.942 & 22.21 & $a b$ & -0.038 \\
\hline V48 & 3159 & $11: 13: 24.68$ & 22:08:37.9 & 0.70922 & 0.599 & 22.24 & $a b$ & -0.056 \\
\hline V49 & 2322 & $11: 13: 19.43$ & 22:08:59.4 & 0.76280 & 0.583 & 22.02 & $a b$ & -0.085 \\
\hline V50 & 2736 & 11:13:16.78 & 22:09:08.6 & & & 22.00 & unk & \\
\hline V52 & 2409 & $11: 13: 23.49$ & 22:08:35.1 & 0.55269 & 1.015 & 22.24 & $a b$ & -0.013 \\
\hline V54 & 4201 & $11: 13: 32.64$ & $22: 07: 48.3$ & 0.59702 & 0.711 & 22.26 & $a b$ & 0.001 \\
\hline V55 & 2695 & $11: 13: 27.61$ & 22:08:06.6 & 0.62718 & 1.020 & 22.30 & $a b$ & -0.069 \\
\hline V56 & 4058 & 11:13:28.52 & $22: 08: 01.5$ & 0.34118 & 0.599 & 22.28 & $\mathrm{c}$ & \\
\hline V57 & 2696 & $11: 13: 17.33$ & 22:08:43.8 & 0.42179 & 0.348 & 21.97 & $\mathrm{c}$ & \\
\hline V60 & 4354 & $11: 13: 27.22$ & $22: 07: 51.8$ & 0.60862 & 1.027 & 22.34 & $a b$ & -0.057 \\
\hline V61 & 2712 & $11: 13: 29.18$ & $22: 07: 34.6$ & 0.56883 & 0.810 & 22.20 & $a b$ & 0.007 \\
\hline V62 & 2690 & 11:13:12.35 & 22:08:44.4 & 0.36202 & 0.601 & 22.17 & $\mathrm{c}$ & \\
\hline V64 & 4225 & $11: 13: 21.44$ & $22: 07: 54.2$ & & & 22.29 & unk & \\
\hline V65 & 3354 & 11:13:30.42 & $22: 07: 12.6$ & 0.60716 & 0.686 & 22.21 & $a b$ & -0.002 \\
\hline V67 & 2459 & $11: 13: 17.38$ & $22: 07: 54.3$ & 0.56032 & 1.031 & 22.16 & $a b$ & -0.021 \\
\hline V69 & 3207 & $11: 13: 35.74$ & 22:06:23.9 & & & 22.12 & unk & \\
\hline V70 & 3835 & $11: 13: 27.33$ & $22: 06: 56.8$ & 0.62026 & 0.725 & 22.22 & $a b$ & -0.018 \\
\hline V73 & 3236 & 11:13:13.22 & $22: 07: 48.7$ & & & 22.11 & unk & \\
\hline
\end{tabular}


TABLE I. Continued. RR Lyrae Variables 


\begin{tabular}{|c|c|c|c|c|c|c|c|c|}
\hline Var ID & Star ID & $\begin{array}{c}\mathrm{RA} \\
\mathrm{J} 2000.0\end{array}$ & $\begin{array}{c}\text { DEC } \\
\text { J2000.0 }\end{array}$ & $\begin{array}{l}\text { Period } \\
\text { (Days) }\end{array}$ & $A_{V}$ & $m_{V}$ & $\begin{array}{l}\text { Bailey } \\
\text { Type }\end{array}$ & $\Delta \log P$ \\
\hline V74 & 4029 & $11: 13: 27.42$ & $22: 06: 39.2$ & 0.59392 & 0.854 & 22.26 & $a b$ & -0.019 \\
\hline V75 & 3277 & $11: 13: 31.50$ & $22: 06: 16.6$ & 0.57313 & 1.003 & 22.17 & $\mathrm{ab}$ & -0.027 \\
\hline V76 & 2669 & 11:13:10.62 & $22: 07: 47.1$ & & & 22.06 & unk & \\
\hline V77 & 3611 & 11:13:10.70 & $22: 07: 43.8$ & 0.65676 & 0.569 & 22.18 & $a b$ & -0.018 \\
\hline V78 & 4088 & $11: 13: 20.34$ & $22: 07: 00.0$ & 0.56884 & 0.706 & 22.25 & $a b$ & 0.023 \\
\hline V79 & 3548 & 11:13:29.42 & $22: 06: 08.9$ & 0.63790 & 0.787 & 22.07 & $a b$ & -0.040 \\
\hline V80 & 3875 & $11: 13: 34.82$ & $22: 05: 33.7$ & 0.57016 & 0.944 & 22.10 & $a b$ & -0.015 \\
\hline V81 & 1517 & 11:13:07.99 & $22: 08: 40.7$ & 0.80097 & 0.735 & 21.56 & $a b$ & -0.130 \\
\hline V82 & 1675 & $11: 13: 27.46$ & $22: 07: 27.7$ & 0.39731 & 0.289 & 21.42 & c & \\
\hline V83 & 1770 & $11: 13: 36.13$ & $22: 15: 51.1$ & & & 21.57 & unk & \\
\hline V84 & 1784 & 11:13:43.00 & $22: 07: 26.4$ & 0.60829 & 1.013 & 22.10 & $a b$ & -0.054 \\
\hline V85 & 1900 & 11:13:11.76 & 22:09:13.9 & 0.60888 & 1.080 & 22.08 & $a b$ & -0.065 \\
\hline V86 & 1922 & 11:13:43.72 & $22: 10: 48.4$ & 0.28667 & 0.621 & 21.57 & c & \\
\hline V87 & 1982 & 11:13:33.26 & $22: 10: 23.6$ & 0.53243 & 1.235 & 22.28 & abb & -0.031 \\
\hline V88 & 1996 & 11:13:52.92 & $22: 08: 53.0$ & 0.80806 & 0.546 & 21.74 & $a b$ & -0.105 \\
\hline V89 & 2025 & $11: 13: 48.63$ & $22: 10: 13.3$ & 0.56978 & 1.180 & 22.26 & $a b$ & -0.052 \\
\hline V90 & 2188 & $11: 13: 23.76$ & $22: 15: 28.2$ & 0.55128 & 1.208 & 22.26 & $a b$ & -0.042 \\
\hline V91 & 2230 & $11: 13: 15.20$ & $22: 04: 26.6$ & 0.36791 & 0.532 & 21.93 & $\mathrm{c}$ & \\
\hline V92 & 2269 & $11: 13: 16.10$ & $22: 03: 55.2$ & 0.39916 & 0.467 & 21.99 & c & \\
\hline V93 & 2290 & $11: 13: 44.23$ & $22: 05: 27.8$ & 0.28849 & 0.664 & 22.13 & $\mathrm{c}$ & \\
\hline V94 & 2300 & 11:13:13.55 & $22: 05: 53.4$ & 0.60191 & 0.873 & 22.16 & $a b$ & -0.028 \\
\hline V95 & 2312 & 11:13:39.20 & $22: 07: 15.4$ & 0.64360 & 0.757 & 22.07 & $a b$ & -0.039 \\
\hline V96 & 2334 & 11:13:19.34 & $22: 07: 55.6$ & 0.40767 & 0.517 & 21.98 & c & \\
\hline V97 & 2382 & $11: 13: 40.25$ & $22: 07: 43.3$ & 0.37708 & 0.517 & 22.14 & $\mathrm{c}$ & \\
\hline V98 & 2394 & 11:13:39.79 & $22: 07: 45.7$ & 0.58175 & 0.591 & 22.04 & $a b$ & 0.031 \\
\hline V99 & 2448 & $11: 13: 37.13$ & $22: 13: 44.0$ & 0.54193 & 0.829 & 22.22 & $a b$ & 0.025 \\
\hline
\end{tabular}


TABLE I. Continued. RR Lyrae Variables 


\begin{tabular}{|c|c|c|c|c|c|c|c|c|}
\hline Var ID & Star ID & $\begin{array}{c}\mathrm{RA} \\
\mathrm{J} 2000.0\end{array}$ & $\begin{array}{c}\text { DEC } \\
\text { J2000.0 }\end{array}$ & $\begin{array}{l}\text { Period } \\
\text { (Days) }\end{array}$ & $A_{V}$ & $m_{V}$ & $\begin{array}{l}\text { Bailey } \\
\text { Type }\end{array}$ & $\Delta \log P$ \\
\hline V100 & 2467 & $11: 13: 18.04$ & $22: 07: 58.8$ & 0.51503 & 1.323 & 22.35 & $\mathrm{ab}$ & -0.030 \\
\hline V101 & 2497 & 11:13:49.69 & $22: 08: 35.3$ & 0.60588 & 0.779 & 22.13 & $\mathrm{ab}$ & -0.016 \\
\hline V102 & 2561 & $11: 13: 15.43$ & $22: 10: 09.7$ & 0.52567 & 1.088 & 22.26 & $a b$ & -0.002 \\
\hline V103 & 2593 & $11: 13: 21.09$ & $22: 11: 14.8$ & 0.59649 & 0.997 & 22.25 & $a b$ & -0.043 \\
\hline V104 & 2607 & $11: 13: 40.42$ & $22: 08: 34.6$ & 0.77449 & 0.542 & 22.06 & $a b$ & -0.086 \\
\hline V105 & 2621 & $11: 13: 33.61$ & $22: 04: 11.7$ & 0.53191 & 1.066 & 22.19 & $a b$ & -0.004 \\
\hline V106 & 2633 & $11: 13: 21.18$ & $22: 10: 59.2$ & 0.67730 & 0.403 & 22.05 & $a b$ & -0.006 \\
\hline V107 & 2688 & 11:13:07.32 & 22:07:02.3 & 0.63038 & 1.166 & 22.03 & $a b$ & -0.093 \\
\hline V108 & 2716 & $11: 13: 48.58$ & $22: 15: 11.1$ & 0.62602 & 0.759 & 22.21 & $a b$ & 0.027 \\
\hline V109 & 2767 & $11: 13: 22.05$ & $22: 11: 29.5$ & 0.58815 & 0.872 & 22.19 & $a b$ & -0.018 \\
\hline V110 & 2801 & $11: 13: 44.87$ & $22: 03: 39.7$ & 0.64623 & 1.130 & 22.25 & $a b$ & -0.099 \\
\hline V111 & 2820 & $11: 13: 28.25$ & 22:07:08.2 & 0.28828 & 0.576 & 22.02 & c & \\
\hline V112 & 2830 & $11: 13: 14.64$ & $22: 09: 38.2$ & 0.34577 & 0.754 & 22.32 & c & \\
\hline V113 & 2871 & $11: 13: 33.23$ & $22: 12: 45.3$ & 0.67845 & 0.564 & 22.17 & $a b$ & -0.032 \\
\hline V114 & 2887 & $11: 13: 49.59$ & $22: 04: 44.7$ & 0.64921 & 0.709 & 22.03 & $a b$ & -0.035 \\
\hline V115 & 2896 & $11: 13: 29.42$ & $22: 08: 23.6$ & 0.66809 & 0.530 & 22.11 & $\mathrm{ab}$ & -0.020 \\
\hline V116 & 2899 & $11: 13: 30.10$ & $22: 01: 49.9$ & 0.61526 & 1.214 & 22.08 & $a b$ & -0.090 \\
\hline V117 & 2906 & $11: 13: 35.27$ & $22: 07: 34.5$ & 0.64489 & 0.538 & 22.05 & $a b$ & -0.005 \\
\hline V118 & 2943 & $11: 13: 40.84$ & 22:04:01.1 & 0.35405 & 0.559 & 22.18 & c & \\
\hline V119 & 2962 & $11: 13: 24.90$ & $22: 11: 02.5$ & 0.77051 & 0.361 & 22.13 & $\mathrm{ab}$ & -0.055 \\
\hline V120 & 2990 & $11: 13: 15.84$ & $22: 12: 59.7$ & 0.65160 & 0.572 & 22.16 & $a b$ & -0.015 \\
\hline V121 & 3006 & 11:13:36.99 & $22: 08: 59.5$ & 0.62537 & 0.689 & 22.03 & $a b$ & -0.016 \\
\hline V122 & 3031 & $11: 13: 28.43$ & $22: 04: 49.6$ & 0.53380 & 1.008 & 22.21 & $a b$ & 0.003 \\
\hline V123 & 3055 & $11: 13: 46.00$ & $22: 07: 26.9$ & 0.63797 & 0.631 & 22.18 & $a b b$ & -0.015 \\
\hline V124 & 3072 & $11: 13: 42.68$ & $22: 06: 25.4$ & 0.41264 & 0.460 & 22.09 & $\mathrm{c}$ & \\
\hline V125 & 3086 & $11: 13: 39.67$ & $22: 11: 59.1$ & 0.65294 & 0.771 & 22.09 & $a b$ & -0.047 \\
\hline
\end{tabular}


TABLE I. Continued. RR Lyrae Variables 


\begin{tabular}{|c|c|c|c|c|c|c|c|c|}
\hline Var ID & Star ID & $\begin{array}{c}\mathrm{RA} \\
\mathrm{J} 2000.0\end{array}$ & $\begin{array}{c}\text { DEC } \\
\text { J2000.0 }\end{array}$ & $\begin{array}{l}\text { Period } \\
\text { (Days) }\end{array}$ & $A_{V}$ & $m_{V}$ & $\begin{array}{l}\text { Bailey } \\
\text { Type }\end{array}$ & $\Delta \log P$ \\
\hline V126 & 3121 & $11: 13: 20.53$ & $22: 07: 13.3$ & 0.58798 & 0.882 & 22.17 & $\mathrm{ab}$ & -0.019 \\
\hline V127 & 3122 & $11: 13: 03.71$ & $22: 05: 51.8$ & 0.37047 & 0.580 & 22.15 & c & \\
\hline V128 & 3166 & 11:13:30.67 & $22: 09: 12.5$ & 0.62768 & 0.857 & 22.10 & $a b$ & -0.043 \\
\hline V129 & 3180 & $11: 13: 30.03$ & $22: 05: 22.6$ & 0.54800 & 1.279 & 22.16 & $a b$ & -0.050 \\
\hline V130 & 3218 & $11: 13: 19.76$ & $22: 06: 16.2$ & & & 22.12 & unk & \\
\hline V131 & 3231 & $11: 13: 06.56$ & 22:02:41.1 & 0.63519 & 0.889 & 22.11 & $a b$ & -0.054 \\
\hline V132 & 3240 & $11: 13: 36.35$ & 22:09:04.3 & 0.32976 & 0.444 & 22.26 & $\mathrm{c}$ & \\
\hline V133 & 3257 & $11: 13: 40.16$ & $22: 06: 16.4$ & 0.66382 & 0.638 & 22.09 & $a b$ & -0.034 \\
\hline V134 & 3351 & $11: 13: 11.72$ & $22: 08: 54.0$ & 0.41174 & 0.592 & 22.10 & $\mathrm{c}$ & \\
\hline V135 & 3355 & $11: 13: 48.37$ & $22: 06: 15.5$ & 0.59801 & 0.967 & 22.21 & $a b$ & -0.040 \\
\hline V136 & 3363 & $11: 13: 34.75$ & $22: 07: 44.2$ & 0.58072 & 0.695 & 22.21 & $a b$ & 0.016 \\
\hline V137 & 3419 & $11: 13: 32.00$ & $22: 10: 50.2$ & 0.54721 & 0.725 & 22.23 & $\mathrm{ab}$ & 0.037 \\
\hline V138 & 3470 & $11: 13: 46.51$ & $22: 12: 51.0$ & 0.57274 & 0.789 & 22.18 & $a b$ & 0.007 \\
\hline V139 & 3603 & $11: 13: 39.59$ & $22: 12: 06.3$ & 0.37020 & 0.810 & 22.25 & c & \\
\hline V140 & 3612 & $11: 13: 38.01$ & $22: 06: 10.5$ & 0.57298 & 0.670 & 22.2 & $\mathrm{abb}$ & 0.025 \\
\hline V141 & 3648 & $11: 13: 12.49$ & $22: 04: 50.4$ & 0.53736 & 1.076 & 22.22 & $\mathrm{ab}$ & -0.010 \\
\hline V142 & 3655 & $11: 13: 40.13$ & $22: 10: 37.0$ & 0.57900 & 0.806 & 22.17 & $a b$ & -0.000 \\
\hline V143 & 3668 & $11: 13: 42.93$ & $22: 07: 37.7$ & 0.67390 & 0.687 & 22.16 & $a b$ & -0.048 \\
\hline V144 & 3728 & $11: 13: 43.62$ & $22: 06: 53.2$ & 0.60467 & 0.824 & 22.15 & $\mathrm{ab}$ & -0.022 \\
\hline $\mathrm{V} 145$ & 3753 & $11: 13: 23.40$ & $22: 06: 11.7$ & 0.30380 & 0.442 & 22.14 & c & \\
\hline V146 & 3756 & $11: 13: 10.54$ & $22: 04: 10.3$ & 0.62178 & 0.529 & 22.14 & $a b$ & 0.012 \\
\hline V147 & 3767 & $11: 13: 20.15$ & $22: 05: 44.6$ & 0.39119 & 0.462 & 22.17 & $\mathrm{c}$ & \\
\hline V148 & 3793 & $11: 13: 22.04$ & $22: 10: 54.7$ & 0.59595 & 0.884 & 22.33 & $a b$ & -0.025 \\
\hline V149 & 3819 & $11: 13: 17.59$ & $22: 03: 32.8$ & 0.59440 & 0.980 & 22.20 & $a b$ & -0.039 \\
\hline V150 & 3827 & $11: 13: 44.15$ & $22: 14: 33.2$ & 0.63607 & 0.599 & 22.20 & $a b$ & -0.009 \\
\hline V151 & 3833 & $11: 13: 46.82$ & $22: 09: 44.5$ & 0.64002 & 0.674 & 22.17 & $a b$ & -0.023 \\
\hline
\end{tabular}


TABLE I. Continued. RR Lyrae Variables

\begin{tabular}{c|c|c|c|c|c|c|c|c}
\hline \hline Var ID & Star ID & RA & DEC & Period & $A_{V}$ & $m_{V}$ & Bailey & $\Delta$ logP \\
& & J2000.0 & J2000.0 & (Days $)$ & & & Type & \\
\hline V152 & 3834 & $11: 13: 10.49$ & $22: 07: 59.2$ & 0.39628 & 0.685 & 22.22 & $\mathrm{c}$ & \\
V153 & 3855 & $11: 13: 13.11$ & $22: 06: 31.2$ & 0.53161 & 1.282 & 22.36 & $\mathrm{ab}$ & -0.038 \\
V154 & 3858 & $11: 13: 27.63$ & $22: 07: 37.0$ & 0.57966 & 1.097 & 22.16 & $\mathrm{ab}$ & -0.046 \\
V155 & 3899 & $11: 13: 37.02$ & $22: 10: 09.5$ & 0.57998 & 0.938 & 22.29 & $\mathrm{ab}$ & -0.022 \\
V156 & 3922 & $11: 13: 21.68$ & $22: 12: 57.0$ & 0.66558 & 0.580 & 22.19 & $\mathrm{ab}$ & -0.026 \\
V157 & 3943 & $11: 13: 32.38$ & $22: 07: 20.5$ & 0.70267 & 0.473 & 22.19 & $\mathrm{ab}$ & -0.033 \\
V158 & 3949 & $11: 13: 37.75$ & $22: 09: 35.5$ & 0.38603 & 0.418 & 22.20 & $\mathrm{c}$ & $\mathrm{a}$ \\
V159 & 3970 & $11: 13: 28.80$ & $22: 12: 35.5$ & 0.62165 & 0.648 & 22.18 & $\mathrm{ab}$ & -0.007 \\
V160 & 3989 & $11: 13: 18.88$ & $22: 05: 50.7$ & 0.65722 & 0.477 & 22.16 & $\mathrm{ab}$ & -0.004 \\
V161 & 4055 & $11: 13: 18.78$ & $22: 08: 12.5$ & 0.42312 & 0.508 & 22.16 & $\mathrm{c}$ & \\
V162 & 4127 & $11: 13: 45.68$ & $22: 04: 22.8$ & 0.60893 & 0.711 & 22.33 & $\mathrm{ab}$ & -0.007 \\
V163 & 4147 & $11: 13: 11.63$ & $22: 10: 37.2$ & 0.62955 & 0.633 & 22.31 & $\mathrm{ab}$ & -0.010 \\
V164 & 4159 & $11: 13: 43.33$ & $22: 11: 57.2$ & 0.65348 & 0.859 & 22.19 & $\mathrm{ab}$ & -0.061 \\
V165 & 4165 & $11: 13: 22.33$ & $22: 06: 18.2$ & 0.61594 & 0.709 & 22.17 & $\mathrm{ab}$ & -0.012 \\
V166 & 4200 & $11: 13: 24.30$ & $22: 11: 12.7$ & 0.57983 & 0.879 & 22.33 & $\mathrm{ab}$ & -0.012 \\
\hline V167 & 4238 & $11: 13: 08.79$ & $22: 11: 16.3$ & 0.59477 & 0.789 & 22.32 & $\mathrm{ab}$ & -0.009 \\
\hline
\end{tabular}

TABLE II. DI93 Stars that are non-variable 


\begin{tabular}{c|c|c}
\hline \hline DI93 ID & Star ID & WS Index \\
\hline V3 & 4724 & 1.5 \\
V4 & 3541 & 2.3 \\
V7 & 5486 & 1.1 \\
V12 & 1103 & 1.5 \\
V13 & 4698 & 1.4 \\
V20 & 2365 & 2.8 \\
V26 & 3831 & 1.6 \\
V27 & 5673 & 2.9 \\
V35 & 4612 & 1.2 \\
V42 & 4057 & 1.3 \\
V45 & 3065 & 0.8 \\
V47 & 4421 & 0.9 \\
V51 & 5433 & 1.2 \\
V58 & 4224 & 1.2 \\
V59 & 3939 & 1.2 \\
V63 & 3890 & 2.0 \\
V66 & 1291 & 1.1 \\
V68 & 3558 & 2.7 \\
V71 & 4318 & 2.4 \\
V72 & 3445 & 2.7 \\
\hline
\end{tabular}

TABLE III. Anomalous Cepheids 


\begin{tabular}{c|c|c|c|c|c|c|c|c}
\hline \hline Var ID & Star ID & $\begin{array}{c}\text { RA } \\
\text { J2000.0 }\end{array}$ & $\begin{array}{c}\text { DEC } \\
\text { J2000.0 }\end{array}$ & $\begin{array}{c}\text { Period } \\
\text { (Days) }\end{array}$ & $A_{V}$ & $m_{V}$ & Pulsation Mode & Swope ID \\
\hline V53 & 645 & $11: 13: 22.39$ & $22: 08: 34.6$ & 1.48466 & 1.240 & 20.45 & Fundamental & V27 \\
V170 & 737 & $11: 13: 09.58$ & $22: 10: 26.3$ & 1.37955 & 1.050 & 20.59 & Fundemental & V203 \\
V171 & 1205 & $11: 13: 14.54$ & $22: 11: 41.6$ & 0.39191 & 0.879 & 21.38 & Overtone & V51 \\
V172 & 1545 & $11: 13: 34.75$ & $22: 13: 44.8$ & 0.41907 & 0.850 & 21.70 & Overtone & V1 \\
\hline
\end{tabular}




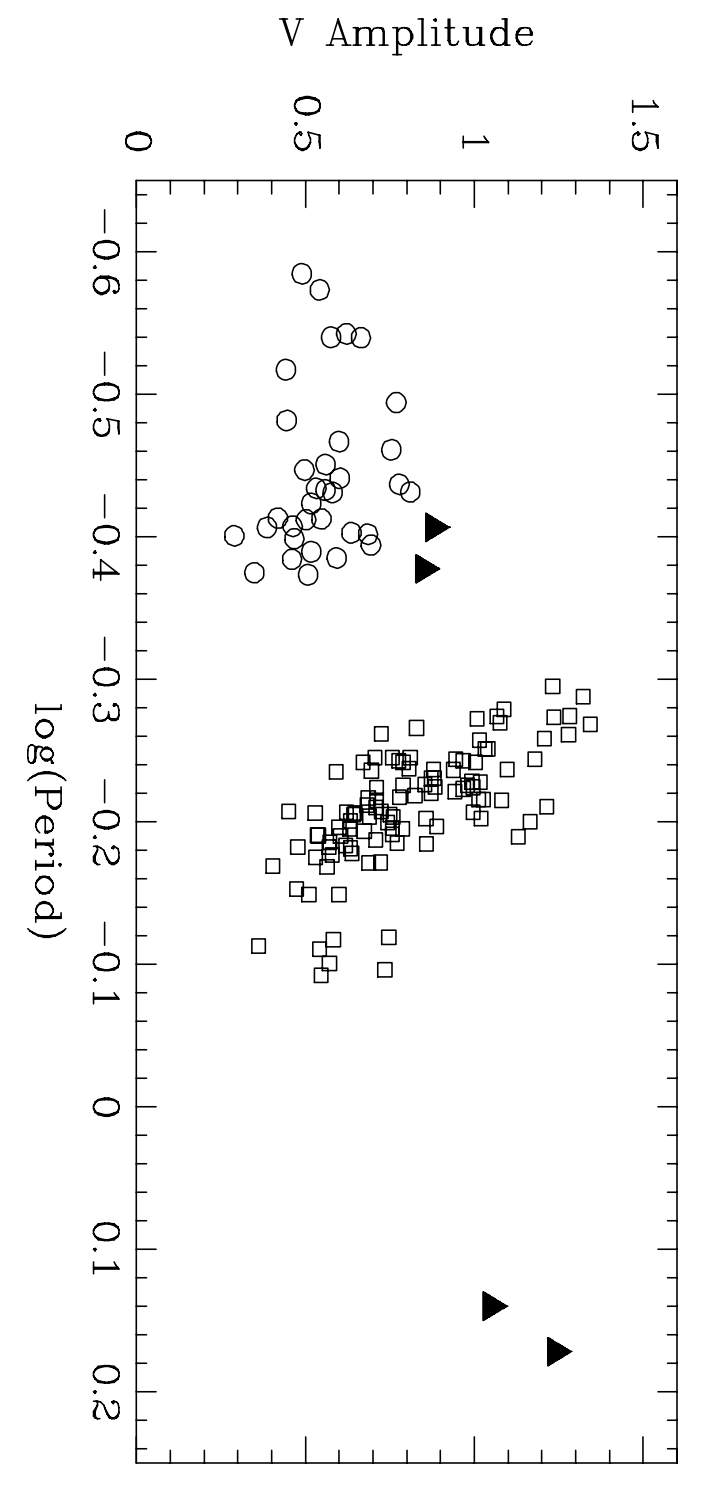




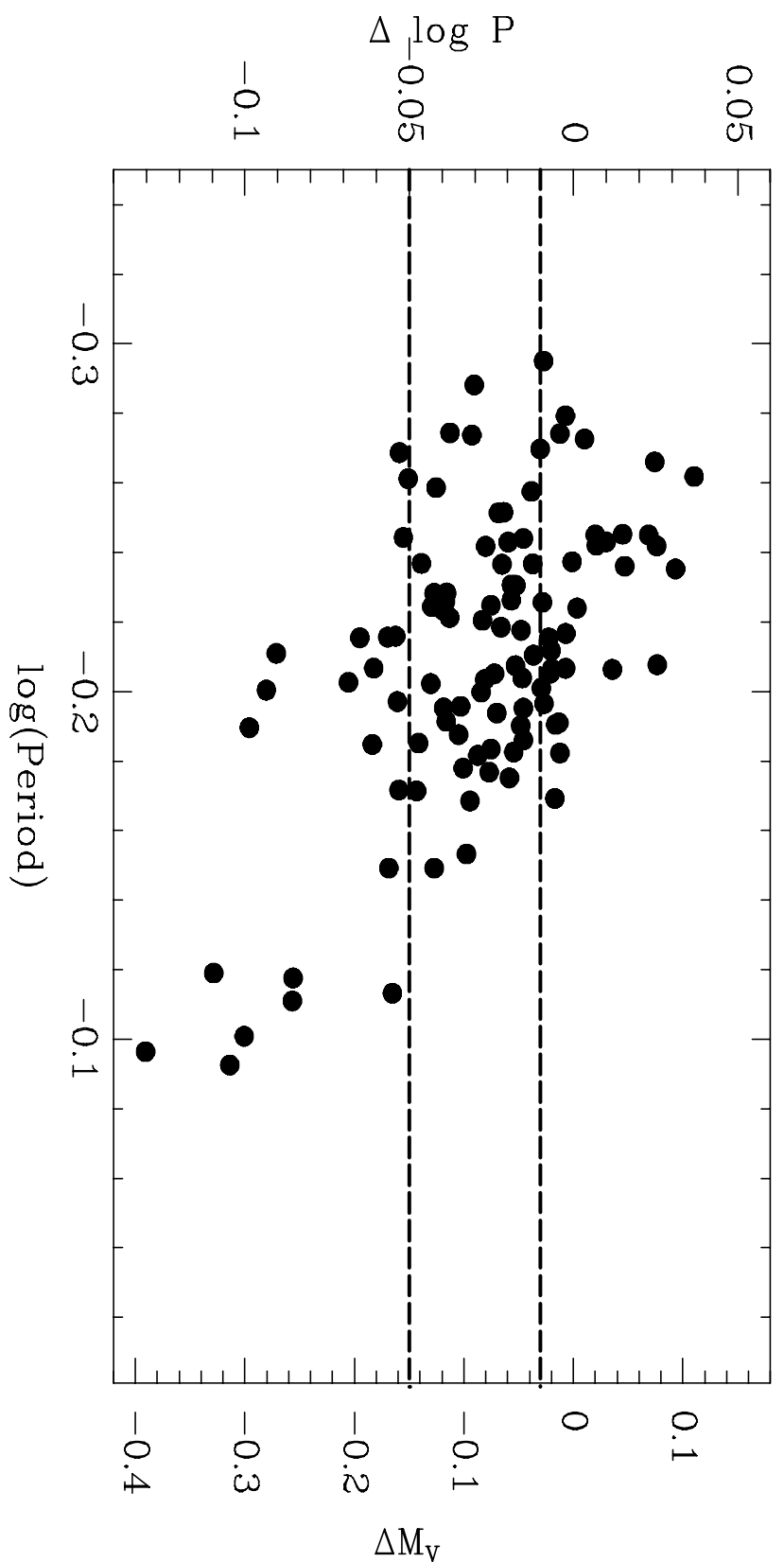




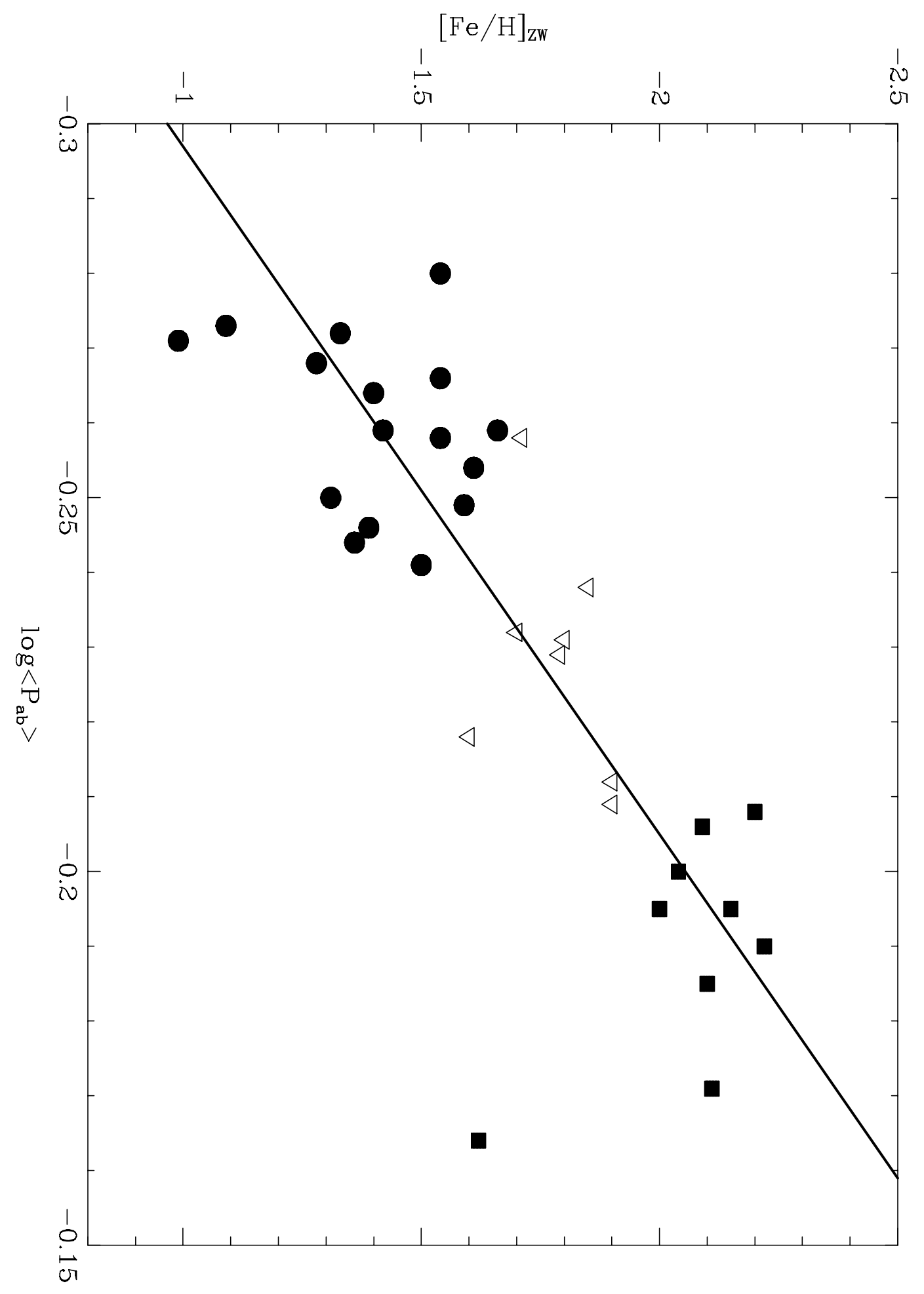



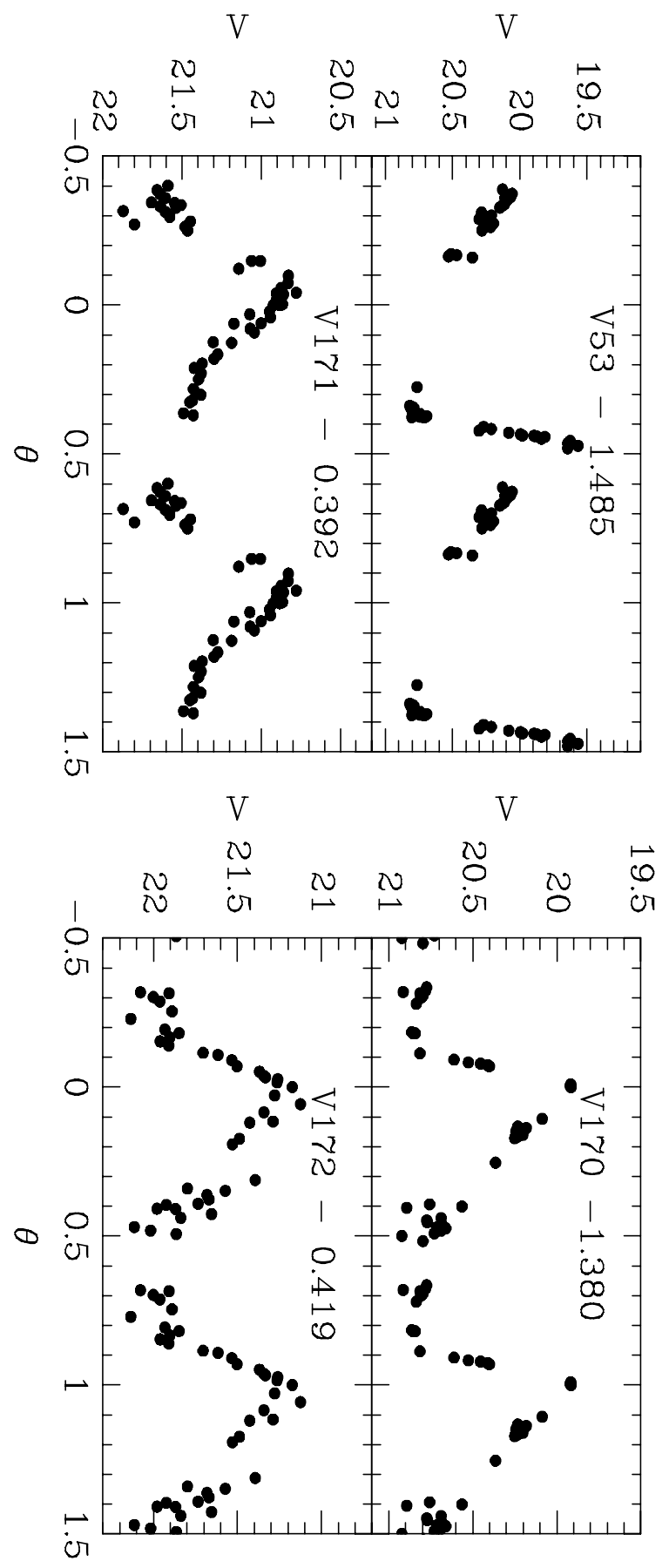


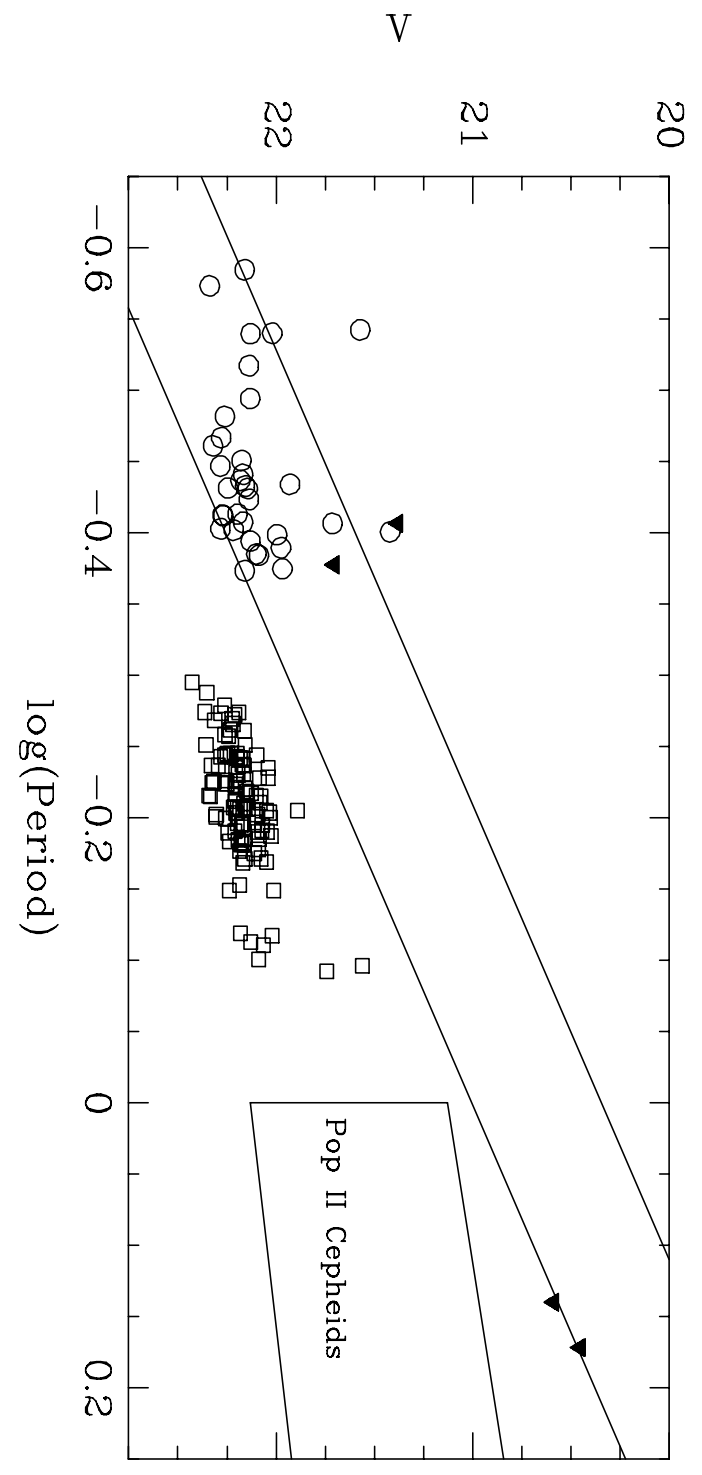


This figure "Siegel.RRfig1.gif" is available in "gif" format from: http://arxiv.org/ps/astro-ph/0004099v1 
This figure "Siegel.RRpage1.gif" is available in "gif" format from: http://arxiv.org/ps/astro-ph/0004099v1 
This figure "Siegel.RRfig2.gif" is available in "gif" format from: http://arxiv.org/ps/astro-ph/0004099v1 
This figure "Siegel.RRpage2.gif" is available in "gif" format from: http://arxiv.org/ps/astro-ph/0004099v1 
This figure "Siegel.RRpage3.gif" is available in "gif" format from: http://arxiv.org/ps/astro-ph/0004099v1 
This figure "Siegel.RRpage4.gif" is available in "gif" format from: http://arxiv.org/ps/astro-ph/0004099v1 
This figure "Siegel.RRpage5.gif" is available in "gif" format from: http://arxiv.org/ps/astro-ph/0004099v1 
This figure "Siegel.RRpage6.gif" is available in "gif" format from: http://arxiv.org/ps/astro-ph/0004099v1 